\title{
Analysis of hydrogen mobility in Nb-based alloy membranes in view of new description of hydrogen permeability based on hydrogen chemical potential.
}

\author{
A. Suzuki ${ }^{\mathrm{a}}$, H. Yukawa ${ }^{\mathrm{a}, *}$, T. Nambu ${ }^{\mathrm{b}}$, Y. Matsumoto ${ }^{\mathrm{c}}$, Y. Murata $^{\mathrm{a}}$ \\ ${ }^{a}$ Department of Materials Science and Engineering, Graduate School of Engineering, Nagoya \\ University, Furo-Cho. Chikusa-ku, Nagoya 464-8603, Japan \\ ${ }^{b}$ Department of Materials Science and Engineering, Suzuka National College of Technology, \\ Shiroko-Cho, Mie 510-0294, Japan \\ ${ }^{\mathrm{c}}$ Department of Mechanical Engineering, Oita National College of Technology, Maki, Oita 870-0152, \\ Japan
}

\section{ABSTRACT}

The hydrogen permeability of Nb-based alloy membranes have been analyzed in view of the new description of hydrogen permeation based on hydrogen chemical potential in order to investigate the alloying effects on the mobility of hydrogen atom, $B$. There is a liner relationship between the normalized hydrogen flux, $J \cdot L$, and the PCT factor, $f_{\text {РСT }}$. The mobility of hydrogen atom is expressed in a format of the Arrhenius equation. Then, the alloying effects on the activation energy, 
$E$, and the pre-exponential factor, $B_{0}$, have been investigated. It is found that logarithm of the pre-exponential factor, $\ln B_{0}$, is proportional to the activation energy, $E$. In other words, when one factor decreases by alloying, the other factor also decreases linearly. As a result, the addition of ruthenium, tungsten and molybdenum into niobium enhances the hydrogen diffusivity at low temperature below the intersection temperature, $T_{\text {int. }}$.

Keywords

Hydrogen permeation, Hydrogen chemical potential, Hydrogen diffusivity,

Pressure-composition-isotherm, Nb-based alloy membrane

* Corresponding author. Tel.: +81 52789 3233; fax: +81 527893233.

E-mail address: hiroshi@nagoya-u.jp (H. Yukawa)

1. Introduction

The hydrogen permeable alloy membranes are important materials for hydrogen purification technologies [1-5]. For example, Pd-based alloy (e.g., Pd-Ag and Pd-Cu alloys) membranes are widely used practically for the separation and purification of high purity hydrogen gas [5]. Recently, a special attention has been directed towards Nb-based alloys, because of their lower cost and higher hydrogen permeability than currently used Pd-based alloys [6-10]. 
For hydrogen permeable membranes, the hydrogen diffusion is generally the rate-limiting process of the total reaction of hydrogen permeation through them. Therefore, the following Fick's diffusion equation is commonly applied to metal membranes to discuss the hydrogen permeation property of them.

$$
J=-D \frac{d c}{d x}
$$

where $D$ is the diffusion coefficient and $d c / d x$ is the gradient for hydrogen concentration.

Combined Eq. (1) with the Sievert's law, $c=K \sqrt{P}$, the hydrogen permeation coefficient, $\phi=D \times K$, is derived and used as a measure to show the hydrogen permeability of metals and alloys, where $K$ is the Sieverts' solubility constant [8].

It is known that the hydrogen diffusion is not always driven by the hydrogen concentration gradient, $d c / d x$. Strictly speaking, the driving force for hydrogen diffusion is the gradient of the hydrogen chemical potential, $d \mu / d x$. Recently, Suzuki et al. have proposed a new description of hydrogen permeability through metal membrane based on hydrogen chemical potential [11]. The diffusion equation based on the chemical potential is expressed as follows,

$$
J=-c B \frac{d \mu}{d x},
$$

where $B$ is the mobility of hydrogen atom. Assuming the following three conditions, Eq (2) can be modified into Eq (3). (1) The hydrogen permeation reaction reaches to the steady state condition, (2) the equilibrium condition is established between gaseous hydrogen and dissolved hydrogen atom on 
the surface of the metal membrane, and (3) the mobility, $B$, is independent of the hydrogen

concentration.

$$
J=\frac{R T B}{2 L} \int_{C_{2}}^{c_{1}} c \frac{d \ln \left(P / P^{0}\right)}{d c} d c=\frac{R T B}{2 L} f_{P C T},
$$

where $R$ is gas constant, $T$ is absolute temperature, $L$ is the membrane thickness, $c_{1}$ and $c_{2}$ are the hydrogen concentrations at the inlet and outlet sides of the membrane, $P$ is applied hydrogen pressure and $P^{0}$ is the standard hydrogen pressure (i.e. 101325Pa). The integral term in Eq (3) is redefined as the PCT factor, $f_{\mathrm{PCT}}$, which can be determined by analyzing the corresponding pressure-composition-isotherm (PCT curve) of the alloy. The validity of Eq (3) has been confirmed by a series of hydrogen permeation test with pure niobium membrane [11].

In this study, the hydrogen permeability of Nb-based alloys have been analyzed in view of Eq (3) in order to investigate the alloying effects on the mobility of hydrogen atom, $B$.

\section{Experimental procedure}

\subsection{Sample preparation}

Nb-5mol\%Ru, Nb-5mol\%W, Nb-5mol\%W-5mol\%Mo, Nb-8mol\%W-8mol\%Mo alloys are melted by using a tri-arc furnace in a purified argon gas atmosphere. The purities of the raw materials used in this study are 99.96 mass\% for niobium and 99.95mass\% for ruthenium, tungsten and molybdenum. According to the Nb-Ru, Nb-W, Nb-Mo and Mo-W equilibrium phase diagrams, all 
these alloys are composed of a single solid solution phase with simple bcc crystal structure, and this is confirmed by XRD profile analysis. As an example, the XRD profile for Nb-8mol\%W-8mol\%Mo alloy is shown in Fig. 1.

For hydrogen permeation tests, the as-cast ingots are cut into disks with about $\phi 12 \mathrm{~mm}$ in diameter and $0.65 \mathrm{~mm}$ in thickness. For comparison, disks of pure niobium are prepared from a pure niobium rod of $\phi 12 \mathrm{~mm}$ in diameter with the purity of 99.96 mass\%. It is cut into disks with the thickness of about $0.65 \mathrm{~mm}$ and they are annealed in a high purity argon gas atmosphere at $1473 \mathrm{~K}$ for $259.2 \mathrm{ks}$.

Both sides of the disk samples are mechanically polished by alumina abrasive papers followed by the final polishing with $0.3 \mu \mathrm{m}$ alumina powders. The final thickness, $L$, of the specimens are approximately $0.48 \sim 0.50 \mathrm{~mm}$. Subsequently, pure palladium of about $200 \mathrm{~nm}$ in thickness is deposited at $573 \mathrm{~K}$ on both sides of the sample surfaces by using an RF magnetron sputtering apparatus.

\subsection{Pressure-composition-isotherms}

The pressure-composition-isotherms (PCT) are measured for Nb-8mol\%W-8mol\%Mo alloys at 673 773 K by using a Sieverts-type apparatus in order to investigate the hydrogen solubility. A small piece of the sample is set into the PCT apparatus and then evacuated. Subsequently, it is heated up to $773 \mathrm{~K}$ and then activated several times prior to the PCT measurements. The PCT curves are 
measured at $673 \sim 773 \mathrm{~K}$ up to about $5 \mathrm{MPa}$

\subsection{Hydrogen permeation test}

The hydrogen permeation tests are performed at $673 \sim 773 \mathrm{~K}$ by the conventional gas permeation method [6]. The disk sample is set into the hydrogen permeation apparatus and then evacuated.

Subsequently, it is heated up to the measuring temperature and high purity hydrogen gas is introduced to both sides of the disk sample. The testing conditions of the temperature as well as the inlet and outlet hydrogen pressures applied in this study are listed in Table 1. The hydrogen fluxes, $J$, permeated through the disk samples are measured by monitoring the pressure change of the reserve tank with known volume. A detailed explanation of the permeation test is given elsewhere [6].

\section{Results and Discussion}

3.1 Analysis of hydrogen flux in view of new description of hydrogen permeability

Figure 2 shows the pressure-composition-isotherms (PCT) for Nb-8mol\%W-8mol\%Mo alloy measured at $673 \mathrm{~K} \sim 773 \mathrm{~K}$. The PCT factor, $\mathrm{f}_{\mathrm{PCT}}$, for each condition listed in Table 1 is estimated by analyzing the corresponding PCT curve shown in Fig. 2 for Nb-8mol\%W-8mol\%Mo alloy and reported in the literature for pure niobium [12], Nb-5mol\%W, Nb-5mol\%Ru and Nb-5mol\%W-5mol\%Mo alloys [13]. 
The steady-state hydrogen flux, $J$, is measured for each condition listed in Table 1 . It is divided by the inverse of the membrane thickness, $1 / L$, in order to estimate the normalized hydrogen flux, $J$ • $L$. It is noted here that the atomic hydrogen flux, mol H m $\mathrm{s}^{-1}$, is evaluated in this study, which is twice as large as the gaseous hydrogen flux, $\mathrm{mol} \mathrm{H}_{2} \mathrm{~m}^{-1} \mathrm{~s}^{-1}$.

Figure 3 shows the correlation between the normalized hydrogen flux, $J \cdot L$, and the PCT factor, $f_{\mathrm{PCT}}$ for (a) pure niobium, (b) Nb-5mol\%W, (c) Nb-5mol\%Ru, (d) Nb-5mol\%W-5mol\%Mo, and (e) Nb-8mol\%W-8mol\%Mo alloys, respectively. There is a liner relationship between the normalized hydrogen flux, $J \cdot L$, and the PCT factor, $f_{\mathrm{PCT}}$, for each system and each temperature. All the lines shown in Fig. 3 cross at the origin, indicating that the hydrogen permeation reaction takes place follows Eq. (3). The slope of the line shown in Fig. 3 corresponds to RTB / 2 in Eq. (3), indicating that the mobility of hydrogen atom, $B$, is almost constant under the conditions tested in this study and independent of the hydrogen pressure or concentration, in good agreement with the assumption. Thus, the new description of hydrogen permeability expressed by Eq. (3) is applicable to not only pure niobium, but also Nb-based alloys.

\subsection{Analysis of mobility for hydrogen diffusion during hydrogen permeation}

The mobility for hydrogen diffusion during hydrogen permeation can be evaluated from the slope of the line shown in Fig. 3. The estimated values of the mobility are summarized in Table 1. It is 
found that the mobility for hydrogen diffusion is larger for Nb-based alloys than pure niobium, especially at low temperature.

Figure 4 shows the Arrhenius plot of the mobility for hydrogen diffusion during hydrogen permeation for pure niobium and Nb-based alloys. As shown in Fig. 4, there is a liner relationship for each system. Thus, the mobility, $B$, for hydrogen diffusion can be expressed in the following Arrhenius equation.

$$
\ln B=\ln B_{0}-\frac{E}{R T},
$$

where $E$ is the activation energy and $B_{0}$ is the pre-exponential factor. The activation energy and the pre-exponential factor of the mobility for hydrogen diffusion are estimated from the straight line for each system shown in Fig.4, and they are summarized in Table 2. It is noted that the lines shown in Fig. 4 seem to cross at certain temperature around $775 \mathrm{~K}$.

Figure 5 shows the correlation between the pre-exponential factor and the activation energy. It is interesting that there is a liner relationship between the logarithm of the pre-exponential factor, $\ln B_{0}$, and the activation energy, $E$, with positive slope. When the value of one factor decreases by alloying, the other factor also decreases linearly, indicating that the ratio of the alloying effects on the activation energy and the pre-exponential factor is almost constant. Therefore, as shown in Fig. 4, a line for a certain alloy (e.g. Nb-5mol\%W) intersects the line for base metal (i.e., pure niobium) at a certain temperature, which is defined in this paper as the intersection temperature, $T_{\text {int. }}$. Here, $T_{\text {int }}$ is 
determined by the balance of the alloying effects on the activation energy and the pre-exponential factor and the slope of the line shown in Fig. 5 corresponds to $1 / R T_{\text {int }}$. Then, the value of $T_{\text {int }}$ for the systems studied in this paper is estimated to be about $780 \mathrm{~K}$.

At higher temperature than $T_{\text {int }}$, the mobility for hydrogen diffusion is expected to be smaller for Nb-based alloys than pure niobium, because the pre-exponential factor is smaller for Nb-based alloys than pure niobium. On the contrary, at lower temperature than $T_{\mathrm{int}}$, the hydrogen mobility is larger for Nb-based alloys than pure niobium because the activation energy for hydrogen diffusion is smaller for Nb-based alloys than pure niobium. Thus, the addition of ruthenium, tungsten and molybdenum into niobium enhances the hydrogen diffusivity at low temperature below $T_{\text {int. }}$. Further investigation will be needed to confirm the intersection temperature, $T_{\text {int }}$, for other systems, and also to understand the alloying effects on the activation energy and the pre-exponential factor of hydrogen mobility for hydrogen diffusion.

\section{Summary}

A new description of the hydrogen permeability based on hydrogen chemical potential through metal membrane has been applied to Nb-based alloys membranes. There is a liner relationship between the normalized hydrogen flux, $J \cdot L$ and the PCT factor, $f_{\mathrm{PCT}}$, for each system and each temperature. Thus, the new description of the hydrogen permeation is applicable to not only pure 
niobium, but also Nb-based alloys.

From the linear relationship between the normalized hydrogen flux, $J \cdot L$ and the PCT factor, $f_{\mathrm{PCT}}$, the mobility, $B$, for hydrogen diffusion during the hydrogen permeation has been estimated at 673 773K. There is linear relationship between the logarithm of the mobility and the inverse of temperature, so the mobility obeys the Arrhenius equation for each system. The pre-exponential factor, $B_{0}$, and the activation energy for hydrogen diffusion, $E$, for each system are estimated by the intercept and the slope of the line in Arrhenius plot. The logarithm of pre-exponential factor, $\ln B_{0}$, is proportional to the activation energy, $E$. In other words, when one factor decreases by alloying, the other factor also decreases linearly. As a result, the lines of the Arrhenius plot for Nb-based alloys intersect the line for pure niobium at a certain temperature, which is defined as the intersection temperature, $T_{\mathrm{int}}$. It is found that the addition of ruthenium, tungsten and molybdenum into niobium enhances the hydrogen diffusivity at low temperature below $T_{\text {int. }}$.

\section{Acknowledgement}

This research was supported in part by CREST, JST and Grant-in-Aid for Scientific Research from JSPS.

\section{Reference}


[1] E. Kikuchi, Catal Today 56 (2000) 97-101.

[2] G. Meunier, JP. Manaud, Int J Hydrogen Energy. 17(8) (1992) 599-602.

[3] S. Tosti, Int J Hydrogen Energy. 28(12) (2003) 1445-1454.

[4] SC. Chen, CY. Caryat Hung, GC. Tua, MH. Rei, Int J Hydrogen Energy. 33(7) (2008) 18801889.

[5] SN. Paglieri, JD. Way, Separation \& Purification Reviews. 31(1) (2002) 1-169.

[6] T. Nambu, N. Shimizu, H. Ezaki, H. Yukawa, M. Morinaga, J Japan Inst Metals. 69(9) (2005) 841-847.

[7] K. Hashi, K. Ishikawa, T. Matsuda, K. Aoki, J Alloys Compd. 425 (2006) 284-290.

[8] SA. Steward, Lawrence Livermore National Laboratory report UCRL-53441. 1983.

[9] RE. Buxbaum, AB. Kinney, Ind Eng Chem Res. 35(2) (1995) 530-537.

[10] H. Yukawa, T. Nambu, Y. Matsumoto, N. Watanabe, GX. Zhang, M. Morinaga, 49(10) (2008) 2202-2207.

[11] A. Suzuki, H Yukawa, T. Nambu, Y. Matsumoto, Y. Murata, Int. J. Hydrogen Energy 39 (2014) 615-619.

[12] E. Veleckis, RK. Edwards, J Phys Chem 73(8) (1969) 683-692.

[13] H. Yukawa, T. Nambu, Y. Matsumoto, Defect Diffusion Forum 312-315 (2011) 506-512. 
Table 1 Temperature and pressure conditions of the hydrogen permeation tests, and the mobility for hydrogen diffusion.

\begin{tabular}{|c|c|c|c|c|}
\hline \multirow{2}{*}{ sample } & \multirow{2}{*}{$\begin{array}{c}\text { Temperature, } \\
T / \mathrm{K} \\
\end{array}$} & \multicolumn{2}{|c|}{ Hydrogen pressure, $P$ / MPa } & \multirow{2}{*}{$\begin{array}{c}\text { Mobility, } \\
B / \mathrm{m}^{2} \mathrm{~s}^{-1} \mathrm{~mol} \mathrm{H} \mathrm{J}\end{array}$} \\
\hline & & inlet & outlet & \\
\hline \multirow{10}{*}{ Pure Nb } & \multirow{2}{*}{773} & 0.030 & \multirow{2}{*}{0.010} & \multirow{2}{*}{$6.58 \times 10^{-13}$} \\
\hline & & 0.020 & & \\
\hline & \multirow{2}{*}{753} & 0.022 & \multirow{2}{*}{0.005} & \multirow{2}{*}{$4.19 \times 10^{-13}$} \\
\hline & & 0.015 & & \\
\hline & \multirow{2}{*}{733} & 0.015 & \multirow{2}{*}{0.005} & \multirow{2}{*}{$2.74 \times 10^{-13}$} \\
\hline & & 0.009 & & \\
\hline & \multirow{2}{*}{713} & 0.010 & \multirow{2}{*}{0.003} & \multirow{2}{*}{$1.88 \times 10^{-13}$} \\
\hline & & 0.005 & & \\
\hline & \multirow{2}{*}{693} & 0.007 & \multirow{2}{*}{0.003} & \multirow{2}{*}{$1.25 \times 10^{-13}$} \\
\hline & & 0.005 & & \\
\hline \multirow{6}{*}{ Nb-5mol\%W } & \multirow{2}{*}{773} & 0.050 & \multirow{2}{*}{0.010} & \multirow{2}{*}{$7.74 \times 10^{-13}$} \\
\hline & & 0.030 & & \\
\hline & \multirow{2}{*}{723} & 0.020 & \multirow{2}{*}{0.005} & \multirow{2}{*}{$3.58 \times 10^{-13}$} \\
\hline & & 0.012 & & \\
\hline & 673 & 0.008 & 0003 & $130 \times 10^{-13}$ \\
\hline & (5/ & 0.006 & 0.005 & $1.30 \times 10$ \\
\hline & & 0.100 & & \\
\hline & 773 & 0.075 & 0.010 & $8.10 \times 10^{-13}$ \\
\hline & & 0.050 & & \\
\hline $\mathrm{Nb}-5 \mathrm{~mol} \% \mathrm{Ru}$ & 723 & 0.050 & 0.010 & $458 \times 10^{-13}$ \\
\hline & 120 & 0.030 & 0.010 & \\
\hline & 673 & 0.020 & 0.005 & $2.36 \times 10^{-13}$ \\
\hline & (5) & 0.010 & 0.005 & \\
\hline & & 0.100 & & \\
\hline & 773 & 0.070 & 0.010 & $7.73 \times 10^{-13}$ \\
\hline & & 0.050 & & \\
\hline & & 0.040 & & \\
\hline Nb-5mol\%W-5mol\%Mo & 723 & 0.030 & 0.010 & $4.58 \times 10^{-13}$ \\
\hline & & 0.020 & & \\
\hline & & 0.016 & & \\
\hline & 673 & 0.013 & 0.004 & $2.61 \times 10^{-13}$ \\
\hline & & 0.010 & & \\
\hline & 773 & 0.250 & 0010 & $725 \times 10^{-13}$ \\
\hline & (5) & 0.100 & 0.010 & \\
\hline Nb-8mol\%W-8mol\%Mo & 723 & 0.100 & 0010 & $608 \times 10^{-13}$ \\
\hline & 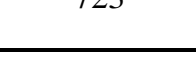 & 0.050 & 0.010 & \\
\hline & 673 & 0.050 & 0.010 & $4.56 \times 10^{-13}$ \\
\hline
\end{tabular}


Table 2 Activation energy and pre-exponential factor of the mobility for hydrogen diffusion in pure niobium and Nb-based alloys.

\begin{tabular}{|c|c|c|c|}
\hline Sample & $\begin{array}{c}\text { Temperature, } \\
T \text { / K }\end{array}$ & $\begin{array}{c}\text { Activation Energy, } \\
\qquad E / \mathrm{kJ}\end{array}$ & $\begin{array}{l}\text { Pre-Exponential factor, } \\
\qquad B_{0} / \mathrm{m}^{2} \mathrm{~s}^{-1} \mathrm{~mol} \mathrm{H} \mathrm{J}\end{array}$ \\
\hline pure $\mathrm{Nb}$ & $693-773$ & 91.5 & $9.58 \times 10^{-7}$ \\
\hline Nb-5mol\%W & \multirow{4}{*}{$673-773$} & 77.4 & $1.36 \times 10^{-7}$ \\
\hline $\mathrm{Nb}-5 \mathrm{~mol} \% \mathrm{Ru}$ & & 53.3 & $3.26 \times 10^{-9}$ \\
\hline Nb-5mol\%W-5mol\%Mo & & 46.9 & $1.14 \times 10^{-9}$ \\
\hline Nb-8mol\%W-8mol\%Mo & & 20.2 & $1.69 \times 10^{-11}$ \\
\hline
\end{tabular}




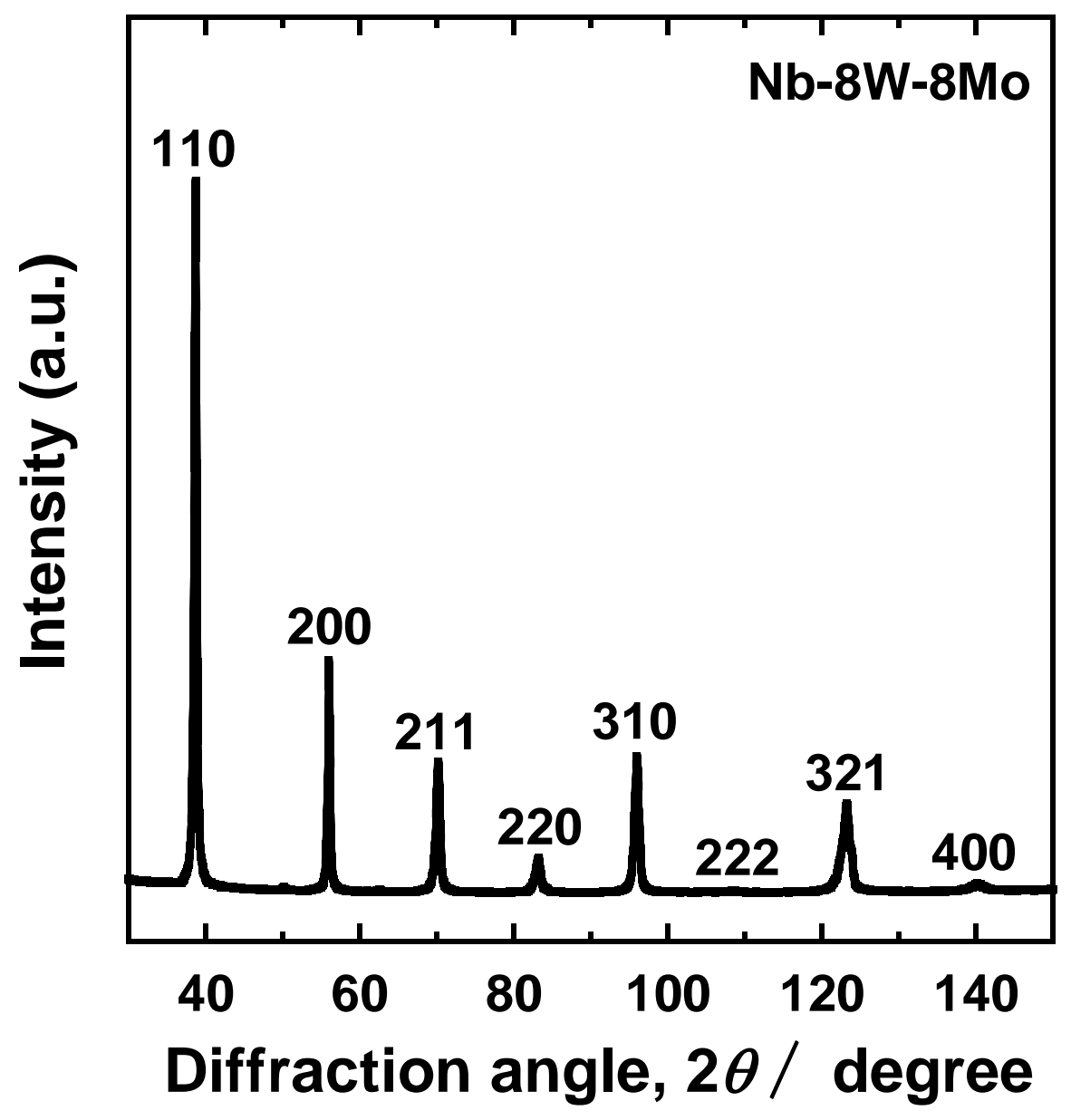

Fig. 1 XRD profile for Nb-8mol\%W-8mol\%Mo alloy. 


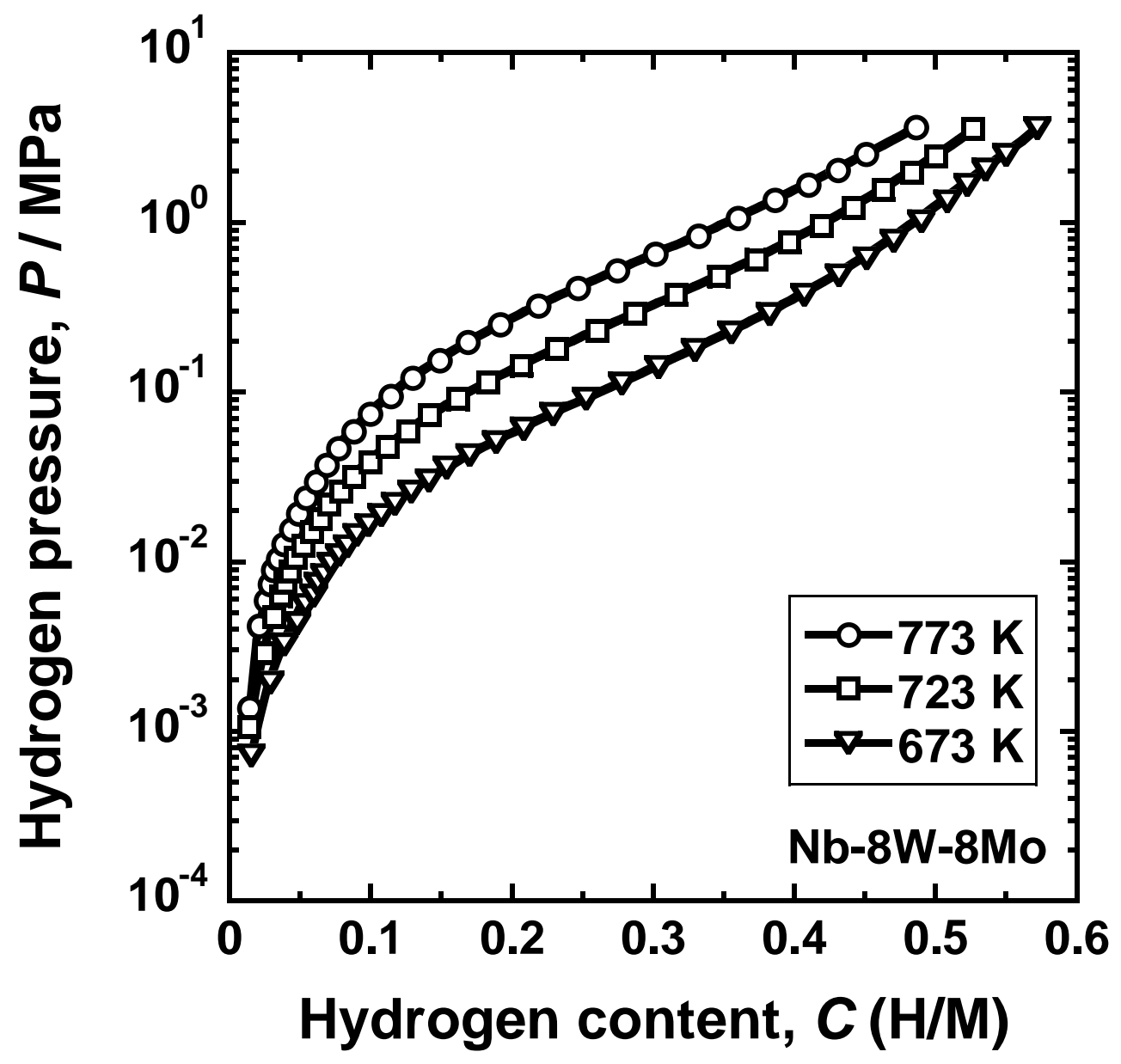

Fig. 2 PCT curve for Nb-8mol\%W-8mol\%Mo alloy measured at 673 773 K. 

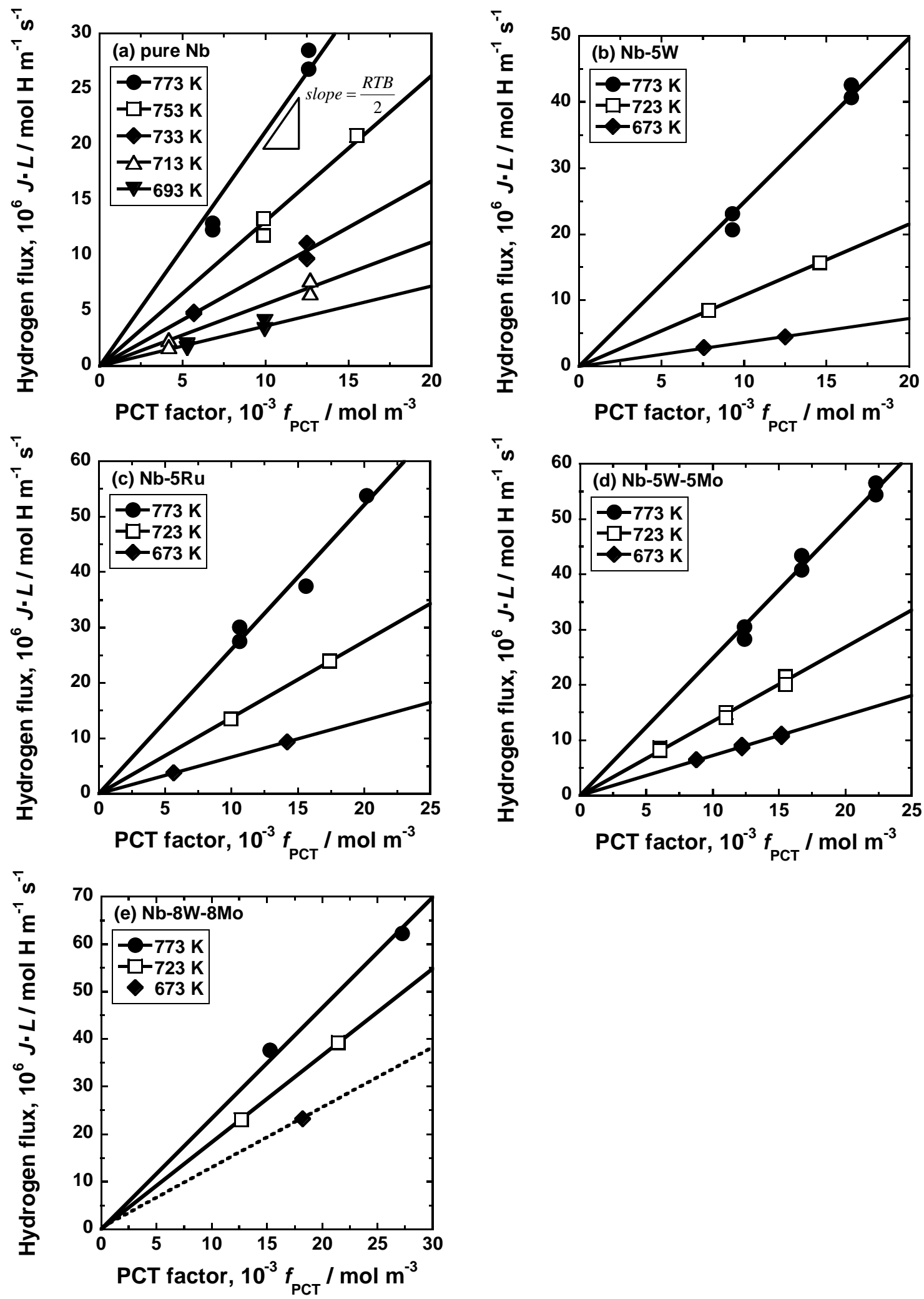

Fig. 3 Correlation between the normalized hydrogen flux, $J \cdot L$, and the PCT factor, $f_{\mathrm{PCT}}$, for (a) pure niobium, (b) Nb-5mol\%W, (c) Nb-5mol\%Ru, (d) Nb-5mol\%W-5mol\%Mo, and (e) Nb-8mol\%W-8mol\%Mo. 


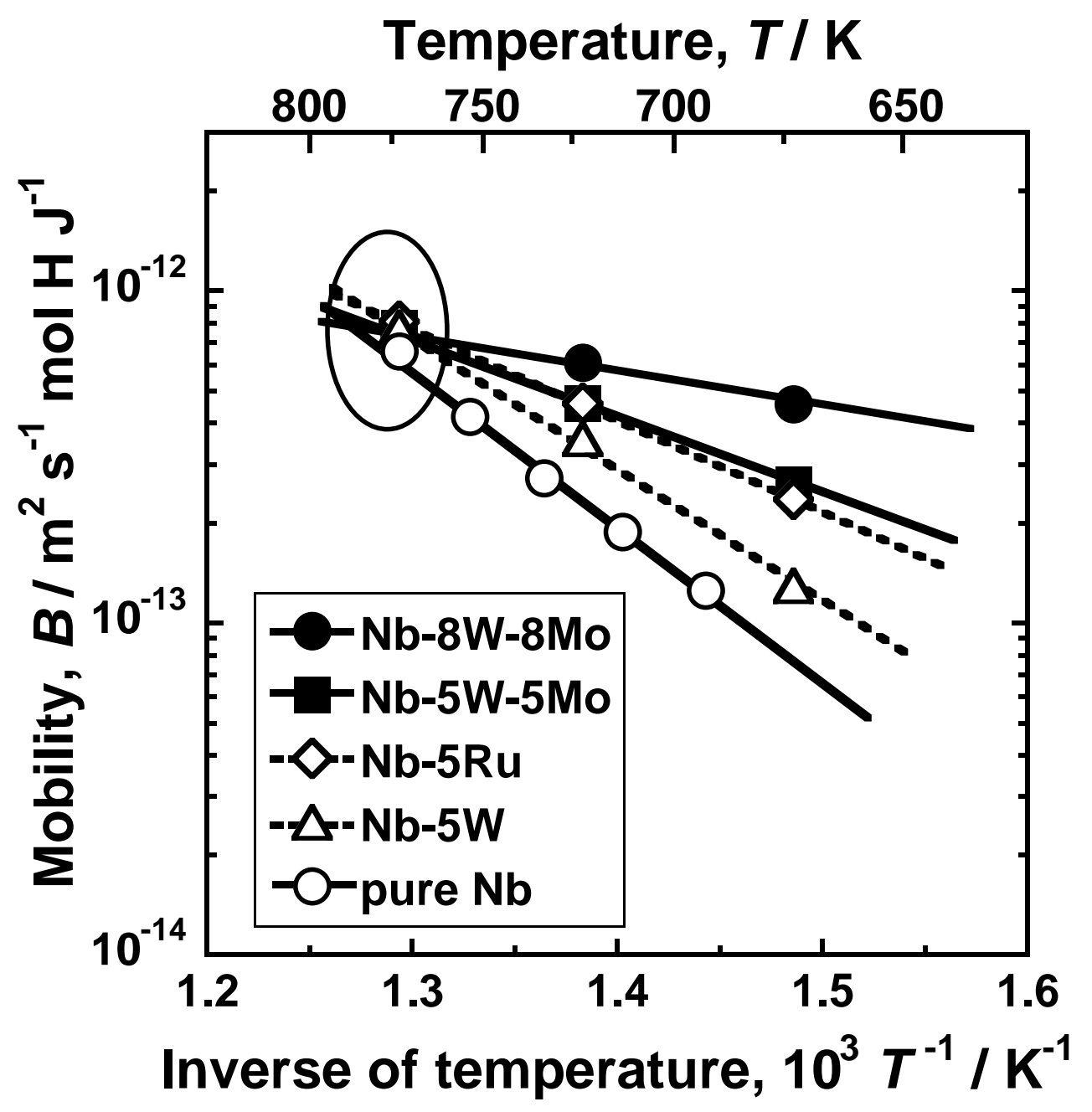

Fig. 4 Arrhenius plots of the mobility for hydrogen diffusion during hydrogen permeation for pure niobium and Nb-based alloys. 


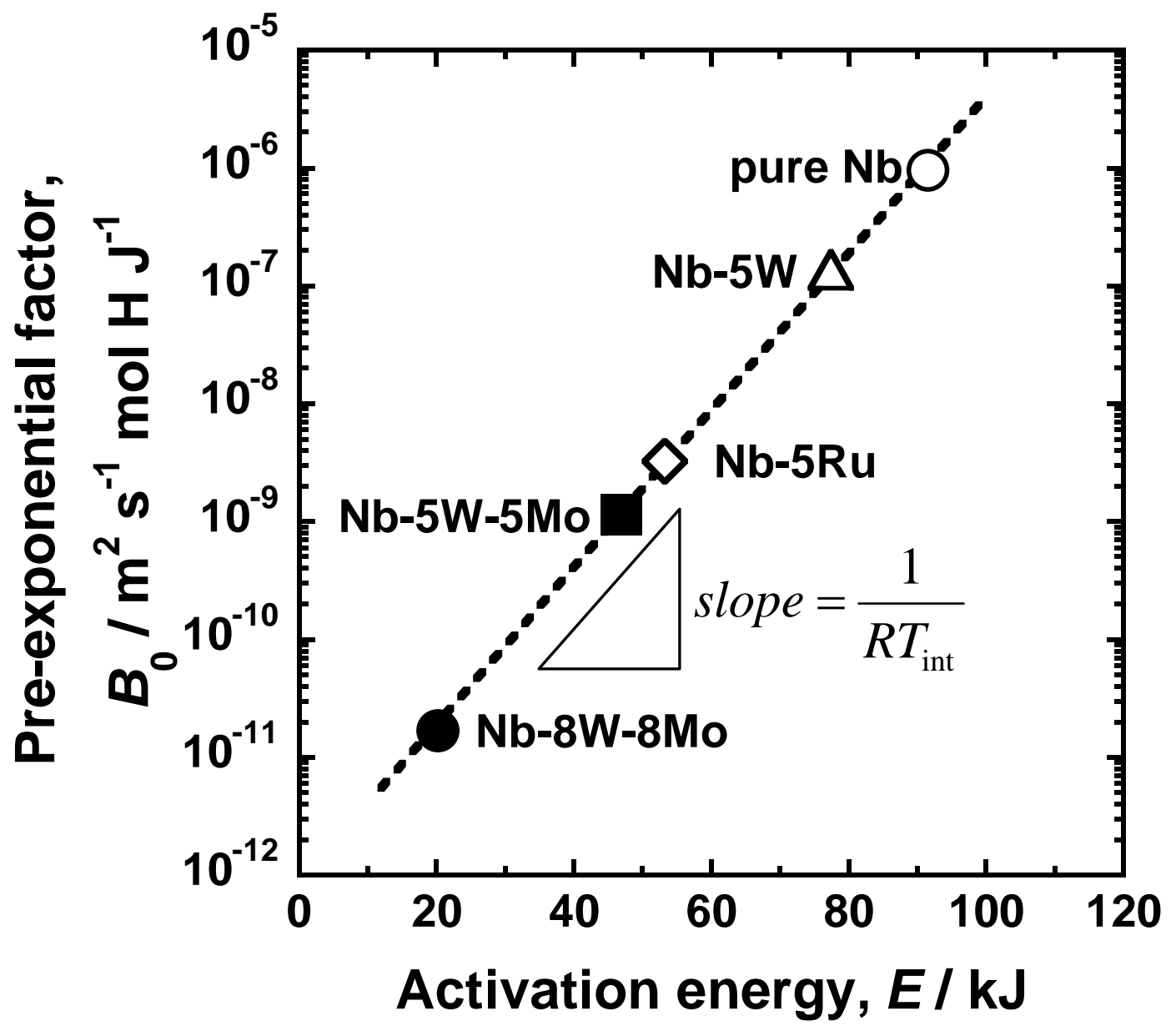

Fig. 5 Correlation between the pre-exponential factor, $B_{0}$, and the activation energy for hydrogen diffusion, $E$. 